\title{
Violência entre Parceiros Íntimos: Uma Comparação dos Índices em Relacionamentos Hetero e Homossexuais
}

\author{
Ana Claudia Ferreira Cezario ${ }^{*}$ \\ Programa de Pós-Graduação em Psicologia da Universidade Federal de Juiz de Fora, \\ Juiz de Fora, MG, Brasil \\ Curso de Psicologia da Universidade Salgado de Oliveira, Juiz de Fora, MG, Brasil \\ Diogo Simões Fonseca \\ Programa de Pós-Graduação em Engenharia Biomédica da Universidade Federal \\ do Rio de Janeiro, Rio de Janeiro, RJ, Brasil \\ Curso de Fisioterapia da Universidade Federal de Juiz de Fora, \\ Governador Valadares, $M G$, Brasil \\ Natalice do Carmo Lopes \\ Universidade Federal de Juiz de Fora, Juiz de Fora, MG, Brasil \\ Núcleo de Estudos em Violência e Ansiedade Social da Universidade Federal \\ de Juiz de Fora, Juiz de Fora, MG, Brasil

\section{Lélio Moura Lourenço} \\ Departamento de Psicologia da Universidade Federal de Juiz de Fora, Juiz de Fora, MG, Brasil \\ Coordenação do Núcleo de Estudos em Violência e Ansiedade Social da Universidade \\ Federal de Juiz de Fora, Juiz de Fora, MG, Brasil
}

\section{Resumo}

A violência entre parceiros íntimos (VPI) pode ser perpetrada por homens e mulheres. Entretanto esse tipo de violência costuma ser mais abordada pela literatura em relacionamentos heterossexuais, apresentando apenas a mulher como vítima e o homem como seu principal agressor. Desta forma, diante das novas configurações sociais e familiares, torna-se importante abordar a violência de modo mais amplo sem restrições de relacionamentos ou de gênero. Neste sentido, este trabalho traz os resultados de duas revisões sistemáticas, uma sobre a VPI em relacionamentos homossexuais e outra sobre a VPI em relacionamentos heterossexuais onde o homem tenha sido vítima de sua respectiva companheira(o). Os resultaram foram obtidos através de análises comparativas e do Teste Qui-quadrado chegando-se a uma amostra total de 196 publicações. Algumas categorias como vítimas, agressores e intervenção apresentaram diferenças significativas com $p$ abaixo de 0,05 . Outras foram comparadas através de estatísticas descritivas, como por exemplo, os principais países de publicação com os Estados Unidos (65) e Brasil (3) na revisão sistemática de VPI contra homens e (19) e (18) respectivamente na revisão da VPI em relacionamento homoafetivos. Propõe-se assim com este trabalho uma análise ampliada da VPI, de forma a contribuir para futuras prevenções e intervenções, independente do tipo de relacionamento ou da opção sexual de seus parceiros.

Palavras-chave: Violência entre parceiros íntimos, violência doméstica, relacionamentos homossexuais, relações homoafetivas.

Endereço para correspondência: Rua Muriaé, 160, Democrata, Juiz de Fora, MG, Brasil 36035-250. E-mail: ana_cfc@yahoo.com.br, simoes81@yahoo.com, natalicecarmo@gmail.com e leliomlourenco@yahoo.com.br Agência de financiamento: Coordenação de Aperfeiçoamento de Pessoal de Nível Superior (CAPES). 


\title{
Intimate Partner Violence: A Comparison of Indices in Hetero and Gay Relationships
}

\begin{abstract}
Intimate partner violence (IPV) may be perpetrated by men and women. However, is more often addressed at heterosexual relationships by literature featuring only women as victims and men as their primary aggressor. Because of this, in the face of new social and families structures, it is important to address violence unrestricted relationships or gender. Thus, this paper presents the results of two systematic reviews, one about the VPI in homosexual relationships and another on IPV in heterosexual relationships which the men have been the victims of their respective wives/girlfriends. The results were obtained through comparative analysis and Chi-square test getting up a full sample of 196 publications. Some categories as victims, aggressors and intervention had presented statistically significant differences with $p$ bellow .05. Others were compared through descriptive statistics, for example, principals publications countries as United States (65) and Brazil (3) in the systematic review of IPV against men and (19) and (18) respectively in the review of IPV in homosexuals relationships. Therefore this work proposes an extended analysis of IPV in order to contribute to future preventions and interventions, regardless of the relationships ways or sexual orientation of its partners.
\end{abstract}

Keywords: Intimate partner violence, domestic violence, gay relationships, relationships in same sex.

\section{Violencia en la Pareja: Una Comparación de los Índices en las Relaciones Heterosexuales y Homosexuales}

\section{Resumen}

La violencia en la pareja puede ser perpetrada por hombres y mujeres. Sin embargo, es más común en las relaciones heterosexuales en las que la mujer es la principal víctima y el hombre agresor. Así, a través de los nuevos perfiles sociales y familiares, es importante para hacer frente a la violencia de manera más amplia, teniendo en cuenta todas las relaciones y orientaciones sexuales. En este sentido, este trabajo presenta datos de dos revisiones sistemáticas, una en el VP en las relaciones homosexuales y el otro sobre la violencia en las relaciones heterosexuales donde el hombre fue agredido por su pareja. El resultado se obtuvo a través del análisis comparativo y la prueba de Chi-cuadrado llegando a una muestra total de 196 publicaciones. Algunas categorías de víctimas, los acosadores y la intervención mostró diferencias significativas con $p$ por debajo de .05. Otros se compararon mediante estadística descriptiva, por ejemplo, los principales países de la publicación con los Estados Unidos (65) y Brasil (3) en la revisión sistemática de la violencia contra los hombres y (19) y (18) respectivamente, en la revisión de La violencia en relación homosexual. Así, se propone en este trabajo un análisis de la violencia ampliada con el fin de contribuir a la prevención y la intervención futura, independientemente del tipo de relación o la orientación sexual de sus parejas.

Palabras clave: Violencia de pareja, la violencia doméstica, las relaciones homosexuales, homoafetivas relaciones.

Etimologicamente, o termo "violência" "provém do latim violentia, relacionado a vis e violare, porta os significados de força em ação, força física, potência, essência, mas também de algo que viola, profana, transgride ou destrói" (Xavier, 2008, p. 21).
Nesta perspectiva, a cartilha "Impacto da Violência na Saúde dos Brasileiros" do Ministério da Saúde (2005) afirma que a violência traz prejuízos individuais e coletivos como danos, lesões, traumas e mortes. Levando a um grave comprometimento orgânico e emocio- 
nal para os indivíduos, social para a população como um todo e econômico, para o sistema de saúde e consequentemente para o país (Ministério da Saúde, 2005). Assim de acordo com o Ministério da Saúde (2005) em decorrência da violência, o Brasil vem apresentando gastos de $10,5 \%$ do seu produto interno bruto; valores que são repassados para atividades terciárias enquanto poderiam estar contribuindo para ações primárias e secundárias de prevenção à violência. Além dos gastos, a literatura aponta também consequências psicológicas da violência como: estresse pós-traumático, depressão, enxaquecas, ansiedade, fobia social, dependência do tabaco, dentre outros (Crane, Hawes, \& Weinberger, 2013; Djikanovic, King, \& Bjegovic-Mikanovic, 2013; Hanby, Fales, Nangle, Serwikand, \& Hedrich, 2012). Comorbidades que podem se desenvolver juntamente às vivências da agressão e que podem motivar as vítimas a irem à busca de assistência; o que evidencia a importância de se estudar mais a temática e de se levantar mais dados em relação às comorbidades como um fator de prevenção à violência.

Uma face da violência, mais específica dos lares e ambientes domésticos, também presente nos dias atuais, é denominada como Violência Doméstica. Esta pode ser caracterizada como qualquer ato/abuso físico, emocional ou sexual de um membro familiar contra o outro, com o objetivo de coibição, por parte do agressor, promovendo uma situação de vulnerabilidade à vítima. Este tipo de violência é definido como "toda ação ou omissão que prejudique o bem-estar, a integridade física, psicológica ou a liberdade e o direito ao pleno desenvolvimento de outro membro da família" (Krug, Dahlberg, Mercy, Zwi, \& Lozano, 2002, p. 15).

Assim, a violência doméstica poderá apresentar como vitimas: crianças, adolescentes, mulheres, idosos e homens. Sendo este último grupo, em função das baixas estatísticas e de alguns dados subnotificados, são pouco mencionados.

Com o intuito de ampliar este tipo de discussão, autores (D’Oliveira et al., 2009; Zaleski, Pinsky, Laranjeira, Ramisetty-Mikler, \& Caetano, 2010) vêm utilizando e estudando o termo IPV - Intimate Partner Violence - ou
Violência por Parceiro Íntimo, cuja definição dada pelo Centers for Disease Control and Prevention (2006 como citado por Cheung, Leung, $\&$ Tsui, 2009, p. 447) é "qualquer abuso físico, emocional ou sexual entre duas pessoas, envolvidas em um relacionamento". Desta forma, esta nova terminologia tem como objetivo apresentar a violência como um resultado da relação entre parceiros íntimos, hetero ou homossexual; e não somente a visão de agressor - vítima/ homem mulher.

No que diz respeito à sua abordagem, a VPI tem sido pesquisada, na maioria dos estudos, em relacionamentos heterossexuais (Durfee, 2011; Zverina, Stam, \& Babins-Wagner, 2011). Assim, ao se comparar os estudos de violência em relacionamentos heterossexuais com as pesquisas em relacionamentos homoafetivos, são bem menores os que falam da VPI entre parceiros de mesmo sexo, tanto em relações femininas quanto masculinas. Por isso a importância de estudos teóricos e discussões no que se refere à temática abordada neste estudo.

Contudo, existem alguns dados já publicados nesta perspectiva homoafetiva ou na perspectiva do homem como vítima de sua respectiva esposa/companheira, que podem ser apresentados (Burke \& Follingstad, 1999; Kuehnle \& Sullivan, 2003; Le Franc, Samms-Vaughan, Hambleton, Fox, \& Brown, 2008; Zaleski et al., 2010). Assim, em 1999 foi realizado nos Estados Unidos, uma revisão crítica de literatura que buscou analisar a prevalência da violência em relacionamentos com parceiros de mesmo sexo (Burke \& Follingstad, 1999). Tal revisão analisou 19 artigos propondo que a taxa de VPI em relacionamentos homoafetivos eram similares às taxas de VPI em relacionamentos heterossexuais. Foram encontrados ainda, mais estudos que se remetiam às relações homossexuais entre mulheres do que em relacionamentos entre homens. Burke e Follingstad (1999) afirmam que tais dados possam ter ocorrido em consequência das teorias e movimentos feministas que existiram em prol da diminuição da violência contra a mulher, sugerindo que a mesma poderia ser mais fraca e estar menos apta a se defender do que o homem. Contudo, o tamanho pequeno da amostra e a data 
de sua publicação pedem novos estudos, maiores e mais atuais, sobre o tema aqui levantado.

Já em 2003, Kuehnle e Sullivan publicaram um artigo, cujo trabalho foi realizado em 1999 em um programa para vítimas de violência através de entrevistas com 262 pessoas que se definiram como homossexuais. Nesta pesquisa foram realizadas entrevistas presenciais e por telefone. Compararam-se dois tipos de violência sofrida: a violência doméstica (cometida entre parceiros íntimos, colegas e/ou familiares) e a violência interpessoal sofrida por preconceito e/ou homofobia. Da amostra entrevistada, 67,3\% afirmaram já terem sido vítimas de violência doméstica enquanto $40 \%$ de preconceitos e homofobia (Kuehnle \& Sullivan, 2003).

No que se refere aos dados já publicados da violência contra o homem perpetrada por sua parceira íntima, Le Franc e colaboradores (2008) realizaram nos países de Barbados, Jamaica e Trinidad e Tobago uma pesquisa com amostra total de 3401 respondentes. As taxas encontradas de violência física por parceiro íntimo contra os homens foram respectivamente, 10,7\%, 13,1\% e $14,8 \%$ da amostra entrevistada. Em relação à violência sexual os dados encontrados foram $0,6 \%, 3,3 \%$ e $1,8 \%$.

No Brasil, em 2005-2006, Zaleski e colaboradores realizaram um estudo transversal com o objetivo de levantar as taxas de violência por parceiro íntimo e suas relações com o abuso de álcool. Da amostra de 1.445 homens e mulheres casados ou vivendo em união estável entrevistados, $10,7 \%$ dos homens já sofreram ou sofriam episódios de violência por suas parceiras, $38,1 \%$ dos homens haviam consumido álcool e $30 \%$ as suas parceiras também (Zaleski et al., 2010).

Neste sentido acredita ser de grande relevância este artigo, cujo objetivo é discutir duas revisões sistemáticas comparando as principais características da violência em relacionamentos homoafetivos e em relações heterossexuais na perspectiva do homem como vítima; tipos de VPI que se comparados à violência contra a mulher, são menos discutidos e estudados. Este estudo poderá ainda contribuir para futuras ações de intervenção às vítimas e agressores da VPI em relacionamentos íntimos, possibilitar o desenvolvimento de ações preventivas na área, além de somar conteúdos para a realização de ações em promoção de saúde de uma forma geral.

\section{Metodologia}

Trata-se de um estudo teórico caracterizado pela comparação de duas revisões sistemáticas: uma relacionada à violência entre parceiros íntimos contra o homem e outra acerca da temática da VPI em relacionamentos homoafetivos. O objetivo do estudo foi discutir semelhanças e divergências entre estes dois tipos específicos de violência no que se refere ao periódico de publicação, país do estudo, principais vítimas e agressores, tipos de agressões mais perpetradas, propostas de intervenção e comorbidades relacionadas.

Para obtenção da amostra foram realizadas duas coletas de dados: uma revisão sistemática do período de 2000 a 2013 sobre a violência contra o homem perpetrada por sua parceira em relacionamentos heterossexuais e outra sobre a VPI presente, especificamente, em relacionamentos homoafetivos também com artigos publicados entre 2000 a 2013.

Em ambos os levantamentos foram utilizadas seis bases de dados: Web of Science, Scopus, Pubmed, Lilacs, Scielo e Dialnet com as terminologias "domestic violence against men", "intimate partner violence against men", "man victim of domestic violence", "adult domestic violence against men", "intimate partner violence by women against men" e "el hombre victima de violencia domestica" para o levantamento da VPI em relacionamentos heterossexuais contra o homem; e "domestic violence in same sex relations*", "intimate partner violence in same sex relations*", "spouse abuse in same sex relations*", "violência doméstica", "violência entre parceiros íntimos" e "violência conjugal" para o levantamento da VPI em relacionamentos homossexuais. As terminologias em inglês foram utilizadas nas bases Web of Science, Pubmed, Scopus e Dialnet enquanto que as em português foram usadas nas bases Lilacs e Scielo.

Após a coleta, realizada separadamente para ambos os levantamentos, seguiram-se as seguintes etapas: (a) exclusão dos artigos repetidos, (b) leitura do título das publicações e exclusão 
daquelas que não se remetiam a pesquisas empíricas, visto ser redundante a análise de revisões, (c) leitura dos resumos das publicações, de forma a aprofundar o tema e; finalmente (d) leitura da introdução das publicações restantes. Esta quarta etapa foi necessária em função de muitos resumos não apresentarem, de forma clara, a população pesquisada, proporcionando-nos dúvidas se realmente se referiam ao tema pesquisado. Após tais etapas foram coletados os artigos que disponibilizavam gratuitamente seus textos completos, chegando-se enfim, à amostra total de 196 publicações, somando-se as duas revisões sistemáticas, para análise das categorias.

O método escolhido para análise dos levantamentos foi o quantitativo através da análise de estatística descritiva e do teste estatístico qui-quadrado. As categorias buscadas na leitura dos textos completos foram: periódico de publicação, país do estudo, principal vítima (se homens ou mulheres), principal agressor (se homens ou mulheres), propostas de intervenção - se sim a direção da mesma, comorbidades, definição de violência usada (violência doméstica, violência entre parceiros íntimos, violência conjugal ou violência intrafamiliar) e tipo de violência abordada (física, psicológica, sexual, patrimonial ou moral).

Para armazenamento dos artigos foi usado um banco de dados através do programa Endnote $\mathrm{X} 5$ e para análise das categorias e construção das tabelas foi usado o programa Microsoft Office Excel 2007.

\section{Resultados}

No que se refere à amostra da VPI em relacionamentos homoafetivos, inicialmente a coleta contou com 1233 artigos, das quais foram excluídos 66 por serem duplicados, totalizando 1167 publicações. Em seguida, foi feito o refinamento com base na análise dos títulos, nesta etapa foram excluídas 459 publicações para posteriormente, após uma nova análise com base nos resumos serem excluídas 250 publicações, restando 458 para análise das introduções dos artigos. A partir desta etapa, foram excluídas as que não mencionavam em suas introduções o interesse pela violência em relacionamentos homoafetivos, sendo por fim, admitidos apenas os artigos disponibilizados gratuitamente chegando-se a uma amostra 74 publicações para serem analisadas.

Já para a amostra de artigos que relatavam pesquisas com homens vítimas da VPI de suas respectivas esposas/companheiras, das seis bases de dados pesquisadas, com publicações pertencentes ao intervalo de 2000 a 2013, foram encontrados a princípio 1666 artigos. Destes, foram excluídos 712 duplicados para depois ser feita uma leitura do título, onde foram excluídos 529 artigos, do resumo 253 artigos, e posteriormente da introdução 23 publicações. Após a busca por artigos disponibilizados gratuitamente foi obtida uma amostra de 111 artigos completos.

Em relação aos resultados do Teste Qui-quadrado, três categorias obtiveram $p$ abaixo de 0,05 com resultados estatisticamente significativos: "país de publicação", "vítimas" e "agressores". Em relação ao país de publicação, como mostra a Tabela 1, o $p$ encontrado foi de 4,2623E-06 ou seja 0,00004 , com destaque para os resultados dos Estados Unidos e Brasil, países com maior número de publicações se comparados aos outros encontrados em ambas revisões. Entretanto, nas duas amostras os Estados Unidos se destacam no que se refere ao número de produções: na amostra da VPI em relacionamentos hetero os Estados Unidos são apontados em 65 estudos enquanto que o Brasil apenas em 3; já na amostra da VPI em relacionamentos homossexuais os Estados Unidos são responsáveis por 19 publicações enquanto que o Brasil aparece com 18 produções. Ao aplicar o teste qui-quadrado, observou-se que tais valores obtidos se divergiram dos valores esperados, demonstrando a diferença estatística entre as amostras, sendo respectivamente 13,1 (Brasil) e 52,3 (Estados Unidos) para a primeira amostra e 7,9 (Brasil) e 31,7 (Estados Unidos) para a segunda amostra. Os outros países demonstrados na Tabela 1 não apresentaram grandes resultados, com frequências de uma publicação cada. Já a subcategoria "outros" foi criada e apresentada nos resultados pelo fato de alguns países terem sido mencionados em apenas uma amostra e vice-versa. Por fim, a subcategoria "não mencionada" se refere aos artigos que não declararam qual território nacional foi realisada a pesquisa. 
Tabela 1

Valores Obtidos e Esperados em Relação ao País de Publicação na Amostra VPI Contra o Homem em Relações Heterossexuais e da Amostra da VPI em Relações Homoafetivas

\begin{tabular}{cccc}
\hline Países & Amostra VPI Homo & Amostra VPI C Homens & Total \\
\hline África do Sul & $1(1,13)$ & $2(1,87)$ & 3 \\
Alemanha & $1(1,13)$ & $2(1,87)$ & 3 \\
Brasil & $18(7,93)$ & $3(13,07)$ & 21 \\
China & $1(0,76)$ & $1(1,24)$ & 2 \\
EUA & $19(31,71)$ & $65(52,29)$ & 84 \\
Não especificado & $18(10,19)$ & $9(16,81)$ & 27 \\
Peru & $1(0,76)$ & $1(1,24)$ & 2 \\
Portugal & $1(0,76)$ & $1(1,24)$ & 2 \\
Suécia & $1(1,51)$ & $3(2,49)$ & 4 \\
Outros Países & $13(18,12)$ & $35(29,88)$ & 48 \\
\hline Total & 74 & 122 & 196 \\
\hline
\end{tabular}

Nota. Os valores entre parênteses equivalem aos valores esperados apresentados pelo teste qui-quadrado.

Quanto às categorias "vítimas" e "agressores", apresentados nas Tabelas 2 e 3 , ambas apresentaram diferenças significativas com $p$ igual a 2,90357E-06 e 0,000157058 respectivamente. As principais vítimas obtidas na amostra de VPI contra homens em relações heterossexuais foram: homens e mulheres (77), homens
(29), mulheres (14) e outras (2), ou seja, publicações que se referiam a vários tipos de vítimas, crianças, adolescentes, idosos; enquanto que na amostra da VPI em relações homossexuais os valores obtidos foram (32), (8), (33) e (1) respectivamente. Na Tabela 2 pode-se observar os valores esperados para cada amostra de acordo com o teste estatístico Qui-quadrado.

Tabela 2

Valores Obtidos e Esperados em Relação às Principais Vítimas Relatadas na Amostra VPI Contra o Homem em Relações Heterossexuais e da Amostra da VPI em Relações Homoafetivas

\begin{tabular}{cccc}
\hline Vítimas & Amostra VPI Homo & Amostra VPI C Homens & Total \\
\hline Homens & $8(13,97)$ & $29(23,03)$ & 37 \\
Homens e mulheres & $32(41,15)$ & $77(67,85)$ & 109 \\
Mulheres & $33(17,74)$ & $14(29,26)$ & 47 \\
Outras & $1(1,13)$ & $2(1,87)$ & 3 \\
\hline Total & 74 & 122 & 196 \\
\hline
\end{tabular}

Nota. Os valores entre parênteses equivalem aos valores esperados apresentados pelo teste qui-quadrado.

Quanto aos agressores, apresentados na Tabela 3, os resultados encontrados na amostra de VPI contra os homens em relações hetero foram: homens e mulheres (77), mulheres (25), homens
(18), geral (2) e não especificado (0); enquanto que na amostra de VPI em relações homossexuais os valores encontrados foram (39), (7), (17), (1) e (10) respectivamente. 
Tabela 3

Valores Obtidos e Esperados em Relação Aos Principais Agressores Relatadas na Amostra VPI Contra o homem em Relações Heterossexuais e da Amostra da VPI em Relações Homoafetivas

\begin{tabular}{cccc}
\hline Agressores & Amostra VPI Homo & Amostra VPI C Homens & Total \\
\hline Geral & $1(1,13)$ & $2(1,87)$ & 3 \\
Homens & $17(13,21)$ & $18(21,79)$ & 35 \\
Homens e Mulheres & $39(43,80)$ & $77(72,20)$ & 116 \\
Mulheres & $7(12,08)$ & $25(19,92)$ & 32 \\
Não especificado & $10(3,78)$ & $0(6,22)$ & 10 \\
\hline Total & 74 & 122 & 196 \\
\hline
\end{tabular}

Nota. Os valores entre parênteses equivalem aos valores esperados apresentados pelo teste qui-quadrado.

No que se refere à categoria "intervenções" através do $p 0,128908$, acima de 0,05 percebe-se que não houve diferença estatística entre as duas amostras. Contudo torna-se importante mencionar aqui os dados que apareceram nos artigos analisados já que em ambas as amostras os resultaram convergiram para a não realização de intervenções, com $73,8 \%$ na amostra da VPI em relações hetero e $60,8 \%$ na de VPI em relações homoafetivas.

Já as categorias "periódicos", "comorbidades" e "destino das intervenções" não puderam ser analisadas pelo qui-quadrado em função da diversidade de dados encontrados. Entretanto serão comparadas através de suas taxas frequenciais de forma a apresentar as principais características das amostras analisadas no que se refere às categorias mencionadas.

Quanto aos periódicos, na amostra da violência contra o homem em relações hetero os que mais se destacaram foram: Journal of Interpersonal Violence (21), Journal of Family Violence
(8), Violence Against Women (4), Aggression and Violent Behavior (3), American Journal of Preventive Medicine (3), Violence and Victims (3) e Journal of Marriage and Family (3); os outros periódicos permaneceram com apenas duas ou uma publicação cada. Já na amostra da VPI em relacionamentos homoafetivos os periódicos de destaque foram Journal of Family Violence com um total de oito publicações, Journal Interpersonal Violence_(5), AIDS Behavior (4) seguidos pelos periódicos Ciência e Saúde Coletiva (3), Caderno de Saúde Pública, Interface Comunicação, Saúde e Educação (3), Investigación y Educación em Enfermería (3) e Violence Against Women (3). Os outros periódicos, assim como na primeira amostra, permaneceram com duas ou uma publicação cada.

Quanto às comorbidades, apresentadas na Tabela 4, em ambas as amostras, o "álcool e as drogas" foram as comorbidades mais citadas sendo destacadas em 29 publicações na primeira amostra e em 16 na segunda. Seguidos pela "depressão", na primeira amostra (10) e na segunda (4).

Tabela 4

Frequência e Frequência Relativa das Comorbidades Apresentadas nas Amostras VPI contra o Homem em Relações Heterossexuais e da VPI em Relações Homoafetivas

\begin{tabular}{ccccc}
\hline Comorbidades & Freq VPI Homo & Freq Relativa & Freq VPI C Homens & Freq Relativa \\
\hline Não cita & 40 & $54,05 \%$ & 70 & $57,38 \%$ \\
Álcool e drogas & 16 & $21,62 \%$ & 29 & $23,77 \%$ \\
Outros & 14 & $18,92 \%$ & 13 & $10,66 \%$ \\
Depressão & 4 & $5,41 \%$ & 10 & $8,20 \%$ \\
\hline Total & 74 & $100,00 \%$ & 122 & $100,00 \%$ \\
\hline
\end{tabular}

Nota. Frequência relativa equivale aos valores em porcentagem. 
Por fim, a última categoria analisada, "destino das intervenções", para a amostra da VPI em relações hetero as frequências relativas foram: vítimas $(43,7 \%)$, população $(18,7 \%)$, vítimas e agressores $(15,6 \%)$, agressores $(3,1 \%)$, profissionais da saúde $(9,4 \%)$ e grupo de risco $(9,4 \%)$; enquanto que para a amostra da VPI em relações homoafetivas foram: vítimas $(55,2 \%)$, população $(20,7 \%)$, grupo de risco $(17,2 \%)$, vítimas e agressores $(6,9 \%)$. As outras subcategorias obtidas por não terem sido citadas em ambas as amostras, também não foram citadas aqui.

Tabela 5

Frequência e Frequência Relativa Referente ao Destino das Intervenções Apresentadas nas Amostras VPI Contra o Homem em Relações Heterossexuais e da VPI em Relações Homoafetivas

\begin{tabular}{cccccc}
\hline Amostra VPI Homo & Freq & Freq Relativa & Destino das Intervenções & Freq & Freq Relativa \\
\hline Vítimas & 16 & $55,17 \%$ & Vítimas & 14 & $43,75 \%$ \\
População & 6 & $20,69 \%$ & População & 6 & $18,75 \%$ \\
Vítimas e Agressores & 2 & $6,90 \%$ & Vítimas e Agressores & 5 & $15,63 \%$ \\
Grupo de risco & 5 & $17,24 \%$ & Grupo de Risco & 3 & $9,38 \%$ \\
& & & Agressores & 1 & $3,13 \%$ \\
& & & Profissionais da Saúde & 3 & $9,38 \%$ \\
\hline Total & 29 & 100 & & 32 & 100 \\
\hline
\end{tabular}

Nota. Frequência relativa equivale aos valores em porcentagem.

\section{Discussão}

Este trabalho teve como objetivo estudar e discutir dois tipos de violência entre parceiros íntimos: a violência contra o homem perpetrada por sua esposa/companheira em relações heterossexuais e a violência entre parceiros íntimos de mesmo sexo, ou seja, em relações homossexuais. Percebe-se que ambas as violências, possuem diferenças em suas especificidades, mas também semelhanças, onde a principal delas é o fato de terem sido pouco discutidas ao longo do tempo se comparadas à violência contra a mulher, principal tipo de VPI encontrada e perpetrada por mais tempo no mundo (Lima, Büchelle, \& Clímaco, 2008).

Contudo, mesmo diante dos altos índices de VPI contra a mulher (Borsoi, Brandão, \& Cavalcanti, 2009; D'Oliveira et al., 2009; Schraiber et al., 2007), torna-se de extrema importância estudar a VPI em todas as suas propriedades sem restrições aos tipos de vítimas ou de relacionamentos.

Em relação aos resultados, na primeira categoria citada, "país de publicação", percebe-se a forte liderança ainda dos Estados Unidos no quesito publicação. Fato que se repete em ambas as amostras, tal resultado não nos é de forma nenhuma surpreendente visto que este país tem apresentado ao longo do tempo numéricas publicações de artigos nas principais bases de dados indexadas, além de seus significativos periódicos bem avaliados e de sua presença na construção de várias linhas teóricas da psicologia. O que chama atenção nesta categoria é o surgimento do Brasil em segundo lugar com 18 publicações na amostra da VPI em relacionamentos homoafetivos, aparecendo como principal articulador nesta temática. O que não ocorre na amostra de VPI em relações hetero contra o homem, no qual o Brasil apareceu apenas com três publicações. Uma hipótese para este resultado pode ser o investimento brasileiro em pesquisas e programas de prevenção à violência contra a mulher juntamente à criação da Lei Maria da Penha (Lei n. $11.340,2006$ ), ainda recente, fatos que podem estar relacionados ao maior interesse em estudar e pesquisar a violência contra a mulher também nas relações homoafetivas.

Nas categorias vítimas e agressores, em ambas as amostras, houve maior destaque para "ho- 
mens e mulheres", sugerindo que ambos podem perpetrar e sofrer a violência independente do tipo de relacionamento que estão inseridos. Resultado que aponta para a nova perspectiva que vem sendo construída através de uma visão bidirecional, ou seja, o entendimento de que a violência pode ser sofrida e perpetrada pelas partes ao mesmo tempo em um relacionamento íntimo em situação de agressão. Alvim e Souza (2005), Bhona (2011), Schraiber, Barros, Couto, Figueiredo, e Albuquerque (2012) e Zaleski e colaboradores (2010) apontam esta perspectiva em seus estudos. Em contra partida, tal resultado também sugere o direcionamento dos autores, ainda em apresentar as mulheres como vítimas, mesmo quando se trata de estudos acerca da violência contra o homem (Cezario \& Lourenço, 2013). Torna-se importante ainda ressaltar, que o fato de se tratar de um assunto subjetivo, como o relacionamento íntimo entre parceiros, e admitir as variáveis que influenciam tais relações como: humor, traços de personalidades, crenças, dentre outros; parece-nos tornar-se pouco preciso apresentar uma única visão de vítima e de agressor.

A terceira categoria apresentada, "intervenções aplicadas", mesmo sem diferenças estatísticas entre as amostras, apresenta um número muito pequeno de publicações que se remetem à aplicação de intervenções. Observa-se que as atenções ainda se voltam para a identificação e levantamento de dados sobre a violência, independente dos parceiros e do tipo de relacionamento. Ainda assim, das poucas publicações que relatam interesse para a intervenção, a maioria se volta ao tratamento da vítima, o que resulta em maiores atividades de atenção terciária e menores ações de prevenção à VPI e promoção da saúde nos relacionamentos.

No que se refere à categoria "periódicos", destacam-se o Journal of Interpersonal Violence e o Journal of Family Violence, revistas que estiveram presentes liderando as duas amostras. Ambas com foco na violência doméstica e intrafamiliar apresentaram publicações de violência entre parceiros íntimos, violência infantil, violência contra o adolescente e contra o idoso. Sendo que o segundo periódico, Journal of Family Violence, possui a peculiaridade de ser interdisciplinar contemplando publicações nas áreas de psicologia, sociologia, psiquiatria, saúde pública, criminologia, direito e serviço social. Características que também justificam sua posição entre os principais periódicos de publicação, encontrados em ambas as amostras.

Quanto às comorbidades citadas nas publicações, tanto na amostra da VPI em relações heterossexuais contra os homens quanto na revisão da VPI em relações homoafetivas, "álcool e drogas", marcaram presença sendo apresentadas como principais comorbidades citadas. Indicando a necessidade de se discutir o papel que estas substâncias vêm assumindo na violência familiar de forma geral (Hines \& Douglas, 2011), já que alguns autores (Minayo \& Deslandes, 1998) defendem que há uma forte associação entre o abuso de álcool e outras drogas com a violência doméstica. Desta forma, o abuso de álcool e outras drogas têm se tornado uma variável de extrema importância no estudo deste tipo específico de violência, tanto para situações em que a pessoa é vítima quanto em situações em que é agressora. Assim, parece ser de fundamental relevância a detecção precoce do abuso de substâncias, já que esta pode ser um componente influente na prevenção da violência. E com tal detecção, desenvolver melhores formas de intervenções, com o objetivo de diminuir índices da VPI, proporcionando uma melhor qualidade de vida para a população em geral.

\section{Conclusão}

Através da análise e comparação entre as amostras da violência entre parceiros íntimos, com o foco no homem como vítima em relacionamentos heterossexuais, e da violência entre parceiros íntimos em relacionamentos homoafetivos, percebe-se que o número de publicações sobre a temática ainda é pequeno e pouco discutido no universo científico, se comparado à violência contra a mulher. Dentro da violência entre parceiros íntimos, tanto em relacionamentos homossexuais quanto em relacionamentos heterossexuais, a mulher ainda aparece como principal vítima, mesmo quando o homem também é citado; evidenciando assim a tendência em colocá-la 
como um ser mais frágil em todos os aspectos considerados, além da forte repercussão que este assunto ainda traz.

Outro ponto marcante observado neste estudo teórico comparativo é a ênfase dada pelos artigos ao puro levantamento de dados, sem preocupações com intervenção e combate à violência, o que evidencia também o pouco interesse dos autores em pensar em alternativas para a diminuição da VPI e no desenvolvimento de atividades de prevenção.

De forma geral, o presente estudo atingiu seu objetivo comparando os trabalhos publicados acerca da violência entre parceiros íntimos com o foco no homem como vítima e a violência entre parceiros íntimos nos relacionamentos homossexuais, apontando divergências e convergências entre ambas as amostras. Evidenciou também que os dois temas ainda são pouco estudados, mas que já há um movimento em relação ao conhecimento e identificação dessa realidade no meio cientifico. Observa-se ainda a necessidade de novos e mais aprofundados estudos sobre a temática, não só das questões teóricas como das questões práticas, estabelecendo assim intervenções e ações a serem realizadas tanto em nível de assistência, como em nível de prevenção e promoção de saúde.

\section{Referências}

Alvim, S. F., \& Souza, L. (2005). Violência conjugal em uma perspectiva correlacional: Homens e mulheres agredidos/agressores. Psicologia: Teoria e Prática, 7(2), 171-206.

Bhona, F. M. C. (2011). Violência doméstica e consumo de álcool entre mulheres: Um estudo transversal por amostragem na cidade de Juiz de Fora - MG (Dissertação de mestrado em Psicologia, Universidade Federal de Juiz de Fora, MG, Brasil).

Borsoi, T. S., Brandão, E. R., \& Cavalcanti, M. L. T. (2009). Ações para o enfrentamento da violência contra a mulher em duas unidades de atenção primária à saúde no município do Rio de Janeiro. Revista Interface - Comunicação Saúde e Educação, 13(28), 165-174. doi:10.1590/ S1414-32832009000100014
Burke, L. K., \& Follingstad, D. R. (1999). Violence in lesbian and gay relationships: Theory, prevalence and correlational factors. Clinical Psychology Review, 19(5), 487-512.

Cezario, A. C. F., \& Lourenço. L. M. (2013). Violência conjugal contra o homem: Uma analise bibliométrica. Gerais: Revista Internacional de Psicologia, 6(1), 144-156.

Cheung, M., Leung, P., \& Tsui, V. (2009). Asian male domestic violence victims: Services exclusive for men. Journal of Family Violence, 24, 447-462.

Crane, C. A., Hawes, S. W., \& Weinberger, A. H. (2013). Intimate partner violence victimization and cigarette smoking: A meta-analytic review. Trauma, Violence and Abuse, 14(4), 305-315. doi:10.1177/1524838013495962

D’Oliveira, A. F. P., Schraiber, L. B., Franca, I. J., Ludermir, A. B., Portella, A. P., Diniz, C. S., ...Valença, O. (2009). Fatores associados à violência por parceiro íntimo em mulheres brasileiras. Revista de Saúde Pública, 43(2), 299-310. doi:10.1590/S0034-89102009005000013

Djikanovic, B., King, E. J., \& Bjegovic-Mikanovic, V. (2013). Gender differences in health symptoms associated with the exposure to physical violence in family: Data from the 2006 National Health Survey in Serbia. Journal of Family Violence, 28, 753-761. doi:10.1007/s10896-0139545-6

Durfee, A. (2011). "Im not a victim, she's an abuser": Masculinity, victimization and protection orders. Gender and Society, 25(3), 316-334. doi:10.1177/0891243211404889

Hanby, M. S. R., Fales, J., Nangle, D. W., Serwikand, A. K., \& Hedrich, U. J. (2012). Social anxiety as a predictor of dating aggression. Journal of Interpersonal Violence, 27(10), 1867-1888. doi:10.1177/0886260511431438

Hines, D. A., \& Douglas, E. M. (2011). Alcohol and drug abuse in men who sustain intimate partner violence. Aggressive Behavior, 38(1), 31-46. doi:10.1002/ab.20418

Krug, E. G., Dahlberg, L. L., Mercy, J. A., Zwi, A. B., \& Lozano, R. (Eds.). (2002). World report on violence and health. Geneva, Switzerland: World Health Organization.

Kuehnle, K., \& Sullivan, S. (2003). Gay and lesbian vitimization: Reporting factors in do- 
mestic violence and bias incidents. Criminal Justice and Behavior, 30(1), 85-96. doi:10.1177/0093854802239164

Le Franc, E., Samms-Vaughan, M., Hambleton, I., Fox, K., \& Brown, D. (2008). Interpersonal violence in three Caribbean countries: Barbados, Jamaica, and Trinidad and Tobago. Revista Panamericana de Salud Publica, 24(6), 409-421. doi:10.1590/S1020-49892008001200005

Lei n. 11.340, de 07 de agosto de 2006. (2006, 08 ago.). Dispõe sobre a coibição da violência doméstica e familiar contra a mulher. Diário Oficial da União. Recuperado em http://www.planalto.gov.br/ccivil_03/_ato2004-2006/2006/lei/ 111340.htm

Lima, D. C., Büchele, F., \& Clímaco, D. A. (2008). Homens, gênero e violência contra a mulher. Saúde e Sociedade, 17(2), 69-81. doi:10.1590/ S0104-12902008000200008

Minayo, M. C. S., \& Deslandes, S. F. (1998). A complexidade das relações entre álcool, drogas e violência. Caderno de Saúde Pública, 14(1), 35 42. doi:10.1590/S0102-311X1998000100011

Ministério da Saúde. (2005). Impacto da violência na saúde dos brasileiros. Recuperado em http:// bvsms.saude.gov.br/bvs/publicacoes/impacto_ violencia.pdf

Schraiber, L. B., Barros, C. R. S., Couto, M. T., Figueiredo, W. S., \& Albuquerque, F. P. (2012). Homens, masculinidade e violência: Estudo em serviços de atenção primária à saúde. Revista Brasileira de Epidemiologia, 15(4), 790-803.
Schraiber, L. B., D’Oliveira, A. F., França, I., Diniz, S., Portella, A. P., Ludermir, A. B., ...Couto, M. T. (2007). Prevalência da violência contra a mulher por parceiro íntimo em regiões do Brasil. Revista de Saúde Pública, 41(5), 797-807. doi:10.1590/s0034-89102007000500014

Xavier, M. (2008). Arendt, Young e humanismo: Um olhar interdisciplinar sobre a violência. Saúde Social, 17(3), 19-32. doi:10.1590/S010412902008000300004

Zaleski, M., Pinsky, I., Laranjeira, R., RamisettyMikler, S., \& Caetano, R. (2010). Intimate partner violence and alcohol consumption. Revista de Saúde Publica, 44(1), 53-59. doi:10.1590/ S0034-89102010000100006

Zverina, M., Stam, H. J., \& Babins-Wagner, R. (2011). Managing victim status in group therapy for men: A discourse analysis. Journal of Interpersonal Violence, 26(14), 2834-2855. doi:10.1177/0886260510390949
Recebido: $12 / 04 / 2013$

$1^{a}$ revisão: $13 / 05 / 2014$

Aceite final: 17/07/2014 